\title{
THE STOCHASTIC LIMIT OF THE OPEN BCS MODEL OF SUPERCONDUCTIVITY
}

\author{
FABIO BAGARELLO
}

Communicated by Syed Twareque Ali

Abstract. We review some recent results concerning the open BCS model of superconductivity as originally proposed by Buffet and Martin. We also briefly analyze some possible generalizations.

\section{Introduction}

In a recent paper, [2], we have analyzed the open BCS model as first proposed in $[6,7]$ using the techniques of the stochastic limit (SL), [1]. Among the other results, we have shown that the same values of the critical temperature and of the order parameters can be found using the SL, in a significantly simpler way. This procedure suggested us to use this approach in order to generalize the original model in the attempt to obtain some control on the critical temperature $T_{c}$. This has been done in [3], where we have discussed the role of a second reservoir in the definition of the model and its consequences on the value of $T_{c}$.

In this paper we review the results of these two papers: in particular, we devote the next section to summarize our results concerning the original model, [2], while in Section 3 we introduce different models with more reservoirs, [3].

\section{The Model}

The model discussed in [2] consists of the system, which is described by means of spin variables, and the reservoir, which is given in terms of bosonic operators. It is contained in a box of volume $V=L^{3}$, with $N$ lattice sites. We define, following $[6,7]$,

$$
H_{N}^{(\mathrm{sys})}=\tilde{\epsilon} \sum_{j=1}^{N} \sigma_{j}^{0}-\frac{g}{N} \sum_{i, j=1}^{N} \sigma_{i}^{+} \sigma_{j}^{-} .
$$


The algebra of the Pauli matrices is given by $\left[\sigma_{i}^{+}, \sigma_{j}^{-}\right]=\delta_{i j} \sigma_{i}^{0},\left[\sigma_{i}^{ \pm}, \sigma_{j}^{0}\right]=$ $\mp 2 \delta_{i j} \sigma_{i}^{ \pm}$. Then, introducing the operators $S_{N}^{\alpha}=\frac{1}{N} \sum_{i=1}^{N} \sigma_{i}^{\alpha}$ and $R_{N}=S_{N}^{+} S_{N}^{-}=$ $R_{N}^{\dagger}, H_{N}^{(\text {sys) }}$ can be simply written as $H_{N}^{(\text {sys })}=N\left(\tilde{\epsilon} S_{N}^{0}-g R_{N}\right)$ and we have

$$
\left[S_{N}^{0}, R_{N}\right]=\left[H_{N}^{(\mathrm{sys})}, R_{N}\right]=\left[H_{N}^{(\mathrm{sys})}, S_{N}^{0}\right]=0
$$

for any given $N>0$. These $S_{N}^{\alpha}$ are all bounded by one in the operator norm, and the commutators $\left[S_{N}^{\alpha}, \sigma_{j}^{\beta}\right]$ go to zero in norm as $\frac{1}{N}$ when $N \rightarrow \infty$, for all $j, \alpha$ and $\beta$.

As for the reservoir, we introduce $N$ bosonic modes $a_{\vec{p}, j}: j=1,2, \ldots, N$, one for each lattice site. $\vec{p}$ is the value of the momentum of the $\mathrm{j}$-th boson which, if we impose periodic boundary conditions on the wave functions, has necessarily the form $\vec{p}=\frac{2 \pi}{L} \vec{n}$, where $\vec{n}=\left(n_{1}, n_{2}, n_{3}\right)$ and $n_{j} \in \mathbb{Z}$. These operators satisfy the following CCR

$$
\left[a_{\vec{p}, i}, a_{\vec{q}, j}\right]=\left[a_{\vec{p}, i}^{\dagger}, a_{\vec{q}, j}^{\dagger}\right]=0, \quad\left[a_{\vec{p}, i}, a_{\vec{q}, j}^{\dagger}\right]=\delta_{i j} \delta_{\vec{p} \vec{q}}
$$

and their free dynamics is given by

$$
H_{N}^{(r e s)}=\sum_{j=1}^{N} \sum_{\vec{p} \in \Lambda_{N}} \epsilon_{\vec{p}} a_{\vec{p}, j}^{\dagger} a_{\vec{p}, j} .
$$

Here $\Lambda_{N}=\left\{\vec{p}=\frac{2 \pi}{L} \vec{n} ; \vec{n} \in \mathbb{Z}^{3}\right\}$. It may be useful to notice that the energy of the different bosons is independent of the lattice site: $\epsilon_{\vec{p}}=\frac{\vec{p}^{2}}{2 m}=\frac{4 \pi^{2}\left(n_{1}^{2}+n_{2}^{2}+n_{3}^{2}\right)}{2 m L^{2}}$.

The interaction between reservoir and system is

$$
H_{N}^{(I)}=\sum_{j=1}^{N}\left(\sigma_{j}^{+} a_{j}(f)+h . c .\right)
$$

where $a_{j}(f)=\sum_{\vec{p} \in \Lambda_{N}} a_{\vec{p}, j} f(\vec{p}), f$ being a given test function which will be later asked to satisfy some regularity conditions. Notice that, in order to keep the notation reasonably simple, we are not using the tensor product symbol here and along this paper, whenever the meaning of the symbols is clear.

The finite volume open system is now described by the following hamiltonian

$$
H_{N}=H_{N}^{0}+\lambda H_{N}^{(I)}, \quad \text { where } \quad H_{N}^{0}=H_{N}^{(\text {sys })}+H_{N}^{(\text {res })}
$$

and $\lambda$ is the coupling constant. 
We have seen in [2] that the free evolution of the interaction hamiltonian, $H_{N}^{(I)}(t)=$ $\mathrm{e}^{\mathrm{i} H_{N}^{0} t} H_{N}^{(I)} \mathrm{e}^{-\mathrm{i} H_{N}^{0} t}$, can be written as

$$
H_{N}^{(I)}(t)=\sum_{j=1}^{N} \sum_{\alpha=0, \pm}\left(\rho_{\alpha}^{j} a_{j}\left(f \mathrm{e}^{\mathrm{i} t \nu_{\alpha}}\right)+h . c\right)
$$

where

$$
\begin{aligned}
& \rho_{0}^{j}=\frac{g^{2} S^{+}}{\omega^{2}}\left(2 S^{-} \sigma_{j}^{+}+S^{0} \sigma_{j}^{0}+2 S^{+} \sigma_{j}^{-}\right) \\
& \rho_{+}^{j}=\frac{g S^{+}}{\omega^{2}}\left(g S^{-} \frac{\omega-g S^{0}}{\omega+g S^{0}} \sigma_{j}^{+}+\frac{\omega-g S^{0}}{2} \sigma_{j}^{0}-g S^{+} \sigma_{j}^{-}\right) \\
& \rho_{-}^{j}=\frac{g S^{+}}{\omega^{2}}\left(g S^{-} \frac{\omega+g S^{0}}{\omega-g S^{0}} \sigma_{j}^{+}-\frac{\omega+g S^{0}}{2} \sigma_{j}^{0}-g S^{+} \sigma_{j}^{-}\right)
\end{aligned}
$$

with

$$
\omega=g \sqrt{\left(S^{0}\right)^{2}+4 S^{+} S^{-}}, \quad \nu=2 \tilde{\epsilon}+g S^{0}
$$

and

$$
\nu_{\alpha}(\vec{p})=\nu-\epsilon_{\vec{p}}+\alpha \omega \text {. }
$$

Here $\alpha$ takes the values $0,+$ and - and $S^{\alpha}=\mathcal{F}-$ strong $\lim _{N \rightarrow \infty} S_{N}^{\alpha} . \mathcal{F}$-strong indicates the strong topology restricted to a certain family $\mathcal{F}$ of relevant states, see [4] and references therein for the details. The introduction of $\mathcal{F}$ is needed because the sequence $S_{N}^{\alpha}$ does not converge in the uniform, strong or even in the weak topology, [4]. As it is widely discussed in, e.g., [1] or [2], the stochastic limit procedure is strongly linked to the result of the following limit, [1],

$$
I(t)=\lim _{\lambda \rightarrow 0} I_{\lambda}(t)=\lim _{\lambda \rightarrow 0}\left(-\frac{\mathrm{i}}{\lambda}\right)^{2} \int_{0}^{t} \mathrm{~d} t_{1} \int_{0}^{t_{1}} \mathrm{~d} t_{2} \omega_{\text {tot }}\left(H_{N}^{(I)}\left(\frac{t_{1}}{\lambda^{2}}\right) H_{N}^{(I)}\left(\frac{t_{2}}{\lambda^{2}}\right)\right)
$$

which turns out to be, [2]

$$
I(t)=-t \sum_{j=1}^{N} \sum_{\alpha=0, \pm}\left\{\omega_{\text {sys }}\left(\rho_{\alpha}^{j} \rho_{\alpha}^{j \dagger}\right) \Gamma_{\alpha}^{(a)}+\omega_{\text {sys }}\left(\rho_{\alpha}^{j \dagger} \rho_{\alpha}^{j}\right) \Gamma_{\alpha}^{(b)}\right\} .
$$

Here $\omega_{\text {tot }}=\omega_{\text {sys }} \otimes \omega_{\beta}$, where $\omega_{\text {sys }}$ is a generic state of the system while $\omega_{\beta}$ is a KMS-state of the reservoir, corresponding to an inverse temperature $\beta$. Notice that we have introduced two complex quantities

$$
\Gamma_{\alpha}^{(a)}=\int_{-\infty}^{0} \mathrm{~d} \tau \sum_{\vec{p} \in \Lambda_{N}}\left|f_{m}(\vec{p})\right|^{2} \mathrm{e}^{-\mathrm{i} \tau \nu_{\alpha}(\vec{p})}, \quad \Gamma_{\alpha}^{(b)}=\int_{-\infty}^{0} \mathrm{~d} \tau \sum_{\vec{p} \in \Lambda_{N}}\left|f_{n}(\vec{p})\right|^{2} \mathrm{e}^{\mathrm{i} \tau \nu_{\alpha}(\vec{p})}
$$


$f_{m}(\vec{p})=\sqrt{m(\vec{p})} f(\vec{p}), f_{n}(\vec{p})=\sqrt{n(\vec{p})} f(\vec{p})$, where $m(\vec{p})$ and $n(\vec{p})$ are the following two points functions: $m(\vec{p})=\omega_{\beta}\left(a_{\vec{p}, j} a_{\vec{p}, j}^{\dagger}\right)$ and $n(\vec{p})=\omega_{\beta}\left(a_{\vec{p}, j}^{\dagger} a_{\vec{p}, j}\right)$. Both $\Gamma_{\alpha}^{(a)}$ and $\Gamma_{\alpha}^{(b)}$ exist because of the standard regularity assumption on $f$ under which the stochastic limit makes sense, $[1,2]$. The analytic expression for $I(t)$ in (10) suggests the introduction of the following stochastic limit hamiltonian, [2]

$$
H_{N}^{(s l)}(t)=\sum_{j=1}^{N} \sum_{\alpha=0, \pm}\left\{\rho_{\alpha}^{j}\left(c_{\alpha j}^{(a)}(t)+c_{\alpha j}^{(b)^{\dagger}}(t)\right)+h . c\right\}
$$

where the operators $c_{\alpha j}^{(\gamma)}(t)$ are assumed to satisfy the commutation rules

$$
\left[c_{\alpha j}^{(\gamma)}(t), c_{\beta k}^{(\mu)^{\dagger}}\left(t^{\prime}\right)\right]=\delta_{j k} \delta_{\alpha \beta} \delta_{\gamma \mu} \delta\left(t-t^{\prime}\right) \Gamma_{\alpha}^{(\gamma)}, \quad \text { for } t>t^{\prime}
$$

The reason for this is that, using the hamiltonian (12) in the computation of

$$
(-\mathrm{i})^{2} \int_{0}^{t} \mathrm{~d} t_{1} \int_{0}^{t_{1}} \mathrm{~d} t_{2} \Omega_{\mathrm{tot}}\left(H_{N}^{(s l)}\left(t_{1}\right) H_{N}^{(s l)}\left(t_{2}\right)\right)
$$

where $\Omega_{\mathrm{tot}}=\omega_{\text {sys }} \otimes \Omega_{\beta}$, and $\Omega_{\beta}$ is a KMS-like state related to the operators $\left\{c_{\alpha j}^{(\gamma)}(t)\right\}$, we recover the same $I(t)$ as in (10), at least if the commutation rules in (13) are satisfied. We refer to [2] and [1] for further details concerning this procedure, and to [5] for a recent review on applications to many-body systems. After few algebraic computations, making use of the so-called time consecutive principle introduced in [1], finally it is possible to associate to this hamiltonian a one parameter group of automorphisms of the observables of the system, representing its time evolution, whose generator $L$, when acting on the intensive operators $S^{0}$ and $S^{+} S^{-}$, looks like

$$
L\left(S^{0}\right):=\mathcal{F}-\text { strong } \lim _{N \rightarrow \infty} L\left(S_{N}^{0}\right)=-\frac{8 g^{4} S^{0}\left(S^{+} S^{-}\right)^{2}}{\omega^{3}} h\left(S^{0}, S^{+} S^{-}\right)
$$

and

$$
L\left(S^{+} S^{-}\right):=\mathcal{F}-\text { strong } \lim _{N \rightarrow \infty} L\left(S_{N}^{+} S_{N}^{-}\right)=-\frac{16 g^{4}\left(S^{+} S^{-}\right)^{3}}{\omega^{3}} h\left(S^{0}, S^{+} S^{-}\right)
$$

where

$$
\begin{aligned}
h\left(S^{0}, S^{+} S^{-}\right)= & \Re \Gamma_{+}^{(a)} \frac{\omega-g}{\left(\omega+g S^{0}\right)^{2}}+\Re \Gamma_{-}^{(a)} \frac{\omega+g}{\left(\omega-g S^{0}\right)^{2}} \\
& +\Re \Gamma_{+}^{(b)} \frac{\omega+g}{\left(\omega+g S^{0}\right)^{2}}+\Re \Gamma_{-}^{(b)} \frac{\omega-g}{\left(\omega-g S^{0}\right)^{2}} .
\end{aligned}
$$


The phase structure of the model is given by the right-hand sides of equations (14) and (15). In particular, it is obtained from the zeros of the functions

$$
f_{1}(x, y)=-\frac{8 g^{4} x y^{2}}{\omega^{3}} h(x, y), \quad f_{2}(x, y)=-\frac{16 g^{4} y^{3}}{\omega^{3}} h(x, y)
$$

where we have introduced, to simplify the notation, $x=S^{0}$ and $y=S^{+} S^{-}$. With this definitions we also have $\omega=g \sqrt{x^{2}+4 y}$ and $\nu=2 \tilde{\epsilon}+g x$. In particular, see [6], the existence of a super-conducting phase corresponds to the existence of a non trivial zero of $f_{1}$ and $f_{2}$, that is, in our scheme, to a non trivial zero of the function $h: h\left(x_{o}, y_{o}\right)=0$. Following Buffet and Martin's original idea, we look for solutions corresponding to $\nu=0$. We will discuss in the next section that this is not the only possibility. This means that, because of (8), the value of $x=S^{0}$ is fixed: $x=-2 \tilde{\epsilon} / g$. This also implies, see [2], that $\Re \Gamma_{-}^{(\gamma)}=0, \gamma=a, b$, while

$$
\Re \Gamma_{+}^{(a)}=\pi \frac{\mathrm{e}^{\beta \omega}}{\mathrm{e}^{\beta \omega}-1} \sum_{\vec{p} \in \mathcal{E}_{N}}|f(\vec{p})|^{2}, \quad \Re \Gamma_{+}^{(b)}=\pi \frac{1}{\mathrm{e}^{\beta \omega}-1} \sum_{\vec{p} \in \mathcal{E}_{N}}|f(\vec{p})|^{2} .
$$

Therefore equation $h\left(x_{o}, y_{o}\right)=0$ becomes

$$
\pi \frac{\mathrm{e}^{\beta \omega}}{\mathrm{e}^{\beta \omega}-1} \sum_{\vec{p} \in \mathcal{E}_{N}}|f(\vec{p})|^{2} \frac{\omega-g}{(\omega+g x)^{2}}+\pi \frac{1}{\mathrm{e}^{\beta \omega}-1} \sum_{\vec{p} \in \mathcal{E}_{N}}|f(\vec{p})|^{2} \frac{\omega+g}{(\omega+g x)^{2}}=0
$$

which produces

$$
\mathrm{e}^{\beta \omega}=\frac{g+\omega}{g-\omega} \quad \text { or equivalently } \quad g \tanh \left(\frac{\beta \omega}{2}\right)=\omega
$$

which is exactly the equation found in [6]. The value of the critical temperature, under which superconductivity takes place, is therefore exactly the same as in [6], $T_{c}:=\frac{g}{2 k}$. It is worth mentioning that also the values of the order parameters coincide with the ones in [6].

The procedure discussed above is technically much simpler than the one used in the original paper, [6]. Among the other simplifications, for instance, a single equation $h(x, y)=0$ must be solved instead of the system $f_{1}(x, y)=f_{2}(x, y)=$ 0 , which is the highly transcendental system appearing in [6].

\section{Generalized Models: More Reservoirs}

In a second paper we have introduced some possible generalizations of the model discussed above which may let $T_{c}$ to increase, [3]. This, we believe, is important in 
Fabio Bagarello

concrete applications, of course, since it would suggest some possible mechanism giving rise to superconductivity at a reasonably high temperature.

The driving idea is very simple and is well put in evidence by the SL approach: suppose that the free evolution of the annihilation operator of the reservoir, $a_{\vec{p}, i}(t)=$ $a_{\vec{p}, i} \mathrm{e}^{-\mathrm{i} \epsilon_{\vec{p}} t}$, is replaced, for some reason, by $a_{\vec{p}, i}(t)=a_{\vec{p}, i} \mathrm{e}^{-\mathrm{i} \gamma \epsilon_{\vec{p}} t}, \gamma$ being some positive constant less than one, $\gamma<1$. As a consequence, the function $\nu_{\alpha}(\vec{p})$ in (9) will be replaced by $\nu_{\alpha}(\vec{p})=\nu-\gamma \epsilon_{\vec{p}}+\alpha \omega$. All the other formulas are left unchanged, at least formally; $h(x, y)$ is the same as in (16), $\Re \Gamma_{-}^{(\rho)}=0, \rho=a, b$, while $\Re \Gamma_{+}^{(a)}=\pi \frac{\mathrm{e}^{\beta \omega / \gamma}}{\mathrm{e}^{\beta \omega / \gamma}-1} \sum_{\vec{p} \in \mathcal{E}_{N}}|f(\vec{p})|^{2}$ and $\Re \Gamma_{+}^{(b)}=\pi \frac{1}{\mathrm{e}^{\beta \omega / \gamma}-1} \sum_{\vec{p} \in \mathcal{E}_{N}}|f(\vec{p})|^{2}$, where, again, $\mathcal{E}_{N}=\left\{\vec{p} \in \Lambda_{N} ; \epsilon_{\vec{p}}=\omega\right\}$. It is easy to check now that equation $h(x, y)=0$ produces, taking $\nu=0$ as before, the following equation

$$
\mathrm{e}^{\beta \omega / \gamma}=\frac{g+\omega}{g-\omega}
$$

which admits a non trivial solution in $] 0, g[$ if $g \beta / \gamma-2>0$, that is under a new critical temperature $T_{c}^{(\gamma)}=\frac{g}{2 k \gamma}=\frac{T_{c}}{\gamma}$, which is larger than $T_{c}$ since $\gamma<1$. This very easy procedure makes the value of the critical temperature to increase leaving unchanged all the physical parameters, in particular $g$. It is worth stressing that a similar mechanism was by no means evident in $[6,7]$.

The main point, therefore, is to find a mechanism which changes the free evolution of the boson operators as shown above. However, since bosons and fermions produce the same free time evolution, a different result could only follow from a reservoir made of quons, see [8] for instance. However this attempt has many technical difficulties and seems not to be the right one.

Another possibility to get a different time evolution for $a_{\vec{p}, i}(t)$ consists in switching on an interaction between the boson reservoir in [2], which we will call $\mathcal{R}_{1}$ and another reservoir, $\mathcal{R}_{2}$, which only interacts with $\mathcal{R}_{1}$ and not with the system $\mathcal{S}$. Within this general scheme we have considered in [3] different choices of the second reservoir, with simple forms of interactions. These choices all share a common output, that is the formal expression of $I(t)$, see equation (10). In particular we have considered the following proposals:

A bosonic second reservoir: The hamiltonian is defined as

$$
H_{N}=H_{N}^{(\mathrm{sys})}+H_{N}^{(\text {res })}+\lambda H_{N}^{(I)}=H_{N}^{0}+\lambda H_{N}^{(I)}
$$

where $H_{N}^{(\mathrm{sys})}$ is given in (1), $H_{N}^{(I)}$ in (4) and

$$
H_{N}^{(r e s)}=H_{N}^{\left(R_{1}\right)}+H_{N}^{\left(R_{2}\right)}+\mu H_{N}^{\left(R_{1}, R_{2}\right)} .
$$


We take $H_{N}^{\left(R_{1}\right)}$ as in (3), $H_{N}^{\left(R_{1}\right)}=\sum_{j=1}^{N} \sum_{\vec{p} \in \Lambda_{N}} \epsilon_{\vec{p}} a_{\vec{p}, j}^{\dagger} a_{\vec{p}, j}$, and

$$
H_{N}^{\left(R_{2}\right)}=\sum_{j=1}^{N} \sum_{\vec{p} \in \Lambda_{N}} \epsilon_{\vec{p}} b_{\vec{p}, j}^{\dagger} b_{\vec{p}, j}, \quad H_{N}^{\left(R_{1}, R_{2}\right)}=\sum_{j=1}^{N} \sum_{\vec{p} \in \Lambda_{N}}\left(a_{\vec{p}, j}^{\dagger} b_{\vec{p}, j}+a_{\vec{p}, j} b_{\vec{p}, j}^{\dagger}\right) .
$$

Here both the reservoirs satisfy a bosonic statistic and they are independent:

$$
\left[a_{\vec{p}, i}, a_{\vec{q}, j}^{\dagger}\right]=\left[b_{\vec{p}, i}, b_{\vec{q}, j}^{\dagger}\right]=\delta_{i j} \delta_{\vec{p}, \vec{q}}, \quad\left[a_{\vec{p}, i}^{\sharp}, b_{\vec{q}, j}^{\sharp}\right]=0 .
$$

A fermionic second reservoir: Here we consider a different reservoir $\mathcal{R}_{2}$ and a different interaction between $\mathcal{R}_{1}$ and $\mathcal{R}_{2}$. In particular we assume that $H_{N}^{\left(R_{2}\right)}=$ $\sum_{j=1}^{N} \sum_{p \in \Lambda_{N}} \eta_{\vec{p}} b_{\vec{p}, j}^{\dagger} b_{\vec{p}, j}$, where the operators $b_{\vec{p}, j}$ satisfy the following CAR, $\left\{b_{\vec{p}, j}, b_{\vec{q}, i}^{\dagger}\right\}=\delta_{i j} \delta_{\vec{p}, \vec{q}},\left\{b_{\vec{p}, j}, b_{\vec{q}, i}\right\}=\left\{b_{\vec{p}, j}^{\dagger}, b_{\vec{q}, i}^{\dagger}\right\}=0$, and commute with the $a_{\vec{p}, j}$,s, and

$$
H_{N}^{\left(R_{1}, R_{2}\right)}=\sum_{j=1}^{N} \sum_{\vec{p} \in \Lambda_{N}} a_{\vec{p}, j}^{\dagger} a_{\vec{p}, j} b_{\vec{p}, j}^{\dagger} b_{\vec{p}, j}
$$

The physical difference between this operator and the hamiltonian $H_{N}^{\left(R_{1}, R_{2}\right)}$ in (22), where if a boson $a$ is created then a boson $b$ is annihiled, is clear: here, in fact, $H_{N}^{\left(R_{1}, R_{2}\right)}$ only counts the number of bosons $a$ and fermions $b$. A consequence of this different definition is that, while in the previous model the total number operator $\hat{N}=\hat{N}_{a}+\hat{N}_{b}=\sum_{j, \vec{p} \in \Lambda_{N}} a_{\vec{p}, j}^{\dagger} a_{\vec{p}, j}+\sum_{j, \vec{p} \in \Lambda_{N}} b_{\vec{p}, j}^{\dagger} b_{\vec{p}, j}$ commutes with $H_{N}^{(r e s)}$ even if $\left[H_{N}^{(r e s)}, \hat{N}_{a}\right] \neq 0$ and $\left[H_{N}^{(r e s)}, \hat{N}_{b}\right] \neq 0$, here we have $\left[H_{N}^{(r e s)}, \hat{N}_{a}\right]=\left[H_{N}^{(r e s)}, \hat{N}_{b}\right]=\left[H_{N}^{(r e s)}, \hat{N}\right]=0$.

A spin-like second reservoir: We end this brief excursus considering another model whose structure is close to that of the previous models. The only differences wrt our previous definitions are again in $H_{N}^{\left(R_{2}\right)}$ and $H_{N}^{\left(R_{1}, R_{2}\right)}$. We put

$$
H_{N}^{\left(R_{2}\right)}=\eta \sum_{j=1}^{N} \sum_{p \in \Lambda_{N}} \tau_{\vec{p}, j}^{0}, \quad H_{N}^{\left(R_{1}, R_{2}\right)}=\sum_{j=1}^{N} \sum_{\vec{p} \in \Lambda_{N}} a_{\vec{p}, j}^{\dagger} a_{\vec{p}, j} \tau_{\vec{p}, j}^{0}
$$

where the operators $\tau_{\vec{p}, j}^{k}, k=0, \pm$, satisfy the same algebra of the Pauli matrices $\left[\tau_{\vec{p}, i}^{+}, \tau_{\vec{q}, j}^{-}\right]=\delta_{i j} \delta_{\vec{p} \vec{q}} \tau_{\vec{p}, j}^{0},\left[\tau_{\vec{p}, i}^{ \pm}, \tau_{\vec{q}, j}^{0}\right]=\mp 2 \delta_{i j} \delta_{\vec{p} \vec{q}} \tau_{\vec{p}, j}^{ \pm}$and commute with the $a_{\vec{p}, j}$ 's.

The interpretation is not very different from that of the previous model: $H_{N}^{\left(R_{1}, R_{2}\right)}$ is again a sort of number operator which counts the excitations of both $\mathcal{R}_{1}$ and $\mathcal{R}_{2}$, without creating or annihilating any of them. The major difference between 
these two last models is in the computation of the mean values of the relevant operators of the models on the related KMS states.

The analysis of these models suggested the existence of different solutions of the equation $h(x, y)=0$, corresponding to other values of the critical temperature, and therefore suggesting the existence of superconducting phases at high temperature, at least under very special assumptions on the constants which define the physical models. Further studies along this direction are in progress.

\section{Acknowledgements}

This work has been partially supported by MURST.

\section{References}

[1] Accardi L., Lu Y. and Volovich I., Quantum Theory and its Stochastic Limit, Springer, Berlin, 2002.

[2] Bagarello F., The Stochastic Limit in the Analysis of the Open BCS Model, J. Phys. A 37 (2004) 2537-2548.

[3] Bagarello F., The Role of a Second Reservoir in the Open BCS Model, OSID, in press.

[4] Bagarello F. and Morchio G., Dynamics of Mean Field Spin Models from Basic Results in Abstract Differential Equations, J. Stat. Phys. 66 (1992) 849-866.

[5] Bagarello F., Many-body Applications of the Stochastic Limit: A Review, Rev. Math. Phys., in press.

[6] Buffet E. and Martin P., Dynamics of the Open BCS Model, J. Stat. Phys. 18 (1978) 585-632.

[7] Martin P., Modèles en Mécanique Statistique des Processus Irréversibles, Lecture Notes in Physics, vol. 103, Springer, Berlin, 1979.

[8] Mohapatra R., Infinite Statistics and a Possible Small Violation of the Pauli Principle, Phys. Lett. B 242 (1990) 407-411.

Fabio Bagarello

Dip. di Metodi e Modelli Matematici

Facoltà di Ingegneria, Università di Palermo

Palermo, I-90128, ITALY

E-mail address: bagarell@unipa.it 